\title{
A NOTE ON CHRONIC IRIDO-CYCLITIS With Special Reference to the Sarcoidosis of Boeck
}

\author{
BY \\ Robert Kemp, M.D.(Liverp.), M.R.C.P.(Lond.) \\ RESEARCH ASSISTANT, DEPARTMENT OF MEDICINE. LIVERPOOL \\ UNIVERSITY. DIRECTOR: PROFESSOR HENRY COHEN
}

THIs condition is not one with which the general physician makes much contact, and without specialised ophthalmological knowledge he needs to walk somewhat carefully. But it is a disease of paramount importance to the ophthalmologist and his patient by reason of the obscurity of its aetiology and because treatment is often unable to prevent serious damage to the sight. It should be understood that my remarks are confined to chronic iritis which has either been insidious from the outset or starting with an acute attack has failed to clear up in the usual way. As regards pathogenesis the literature is rarely helpful and often misleading. Syphilis, tuberculosis and focal sepsis are the three main factors indicated in causation but their relative importance is much disputed. As a result of an investigation at the Manchester Royal Eye Hospital Gray Clegg found that in 55 cases the cause was syphilis in 6, tuberculosis in 2 , a septic focus was found in 12 , whereas the cause was quite unknown in 35 (63.6 per cent.).

To take tuberculosis first it is thought to be the basis of chronic irido-cyclitis in a proportion varying from 5 to 50 per cent. according to the views of individual writers-Doggart (1936). An idea of the grounds, or lack of grounds on which the diagnosis may be based can be obtained from a paper by Irons (1931) and the discussion which followed it. It seems that the diagnostic criteria vary somewhat. The absolute diagnosis is established when tubercle bacilli are found on section. Irrefutable clinical evidence is the appearance of tubercles-though this is very rare. When the condition responds to tuberculin treatment it may be presumed that it has been specific. But the presence of healed, latent or sub-clinical tuberculous lesions or a positive tuberculin reaction should, in my view, not be allowed to establish the diagnosis. This type of iritis is very rarely associated with an active lesion-with the exception of miliary spread-so that when strict proof is demanded it will be seen that tuberculosis plays a small part in aetiology. Syphilis undoubtedly is responsible for a few cases-acute, chronic or Herxhiemer reaction. The American figures are in the neighbourhood of 18 per cent., but it must be remembered speaking approximately that while in America 20 per cent. of the coloured and 10 per cent. of the white population 
are syphilitic; in England the corresponding figure is nearer 3 per cent. There is a good deal of difference of opinion as to focal sepsis as a cause of chronic irido-cyclitis though in the acute disease it may be a considerable factor-Copps and McCormick (1937). Doggart's criticism that the thesis has yet to be proved is in keeping with experience in general medicine. Irons and Brown (1923) are prepared to admit that though focal sepsis may be found it may not be causal. And indeed there may be little difference in the incidence of septic foci in these patients and apparently healthy people. Certainly heroic investigation and eradication has quite failed to solve the question of chronic iritis. Doggart mentions the rare condition of heterochromia iridis and the frequency of iritis in menopausal women regarding this as propably endocrine in nature. It is of course a classical observation that herpes zoster ophthalmicus is a cause of subacute iritis. In addition iritis is rarely but irrefutably seen in the following illnesses - typhoid, influenza, malaria, leprosy, brucellosis, diabetes, gout and lastly the association with gonorrhoea is well known. In most of these latter conditions the iritis is likely to be acute rather than chronic.

To this list there is now to be added a new type of chronic iritis, that of the sarcoidosis of Boeck. Briefly this is a disease of an unknown pathogenesis in which sarcoid granulation tissue is deposited in nearly every organ in the body. On section this resembles the epithelioid reaction of hyperplastic tuberculosis but the clinical sequence of these benign deposits builds up a composite picture from which an accurate diagnosis can be made. A good clinical description is to be found in the paper by Scott (1938). The uveal tract is almost invariably, at an earlier or later stage, the seat of these deposits and a chronic inflammatory reaction results. Such a case is well described by Gordon (1937). A woman gave a story of six years' ocular inflammation. There were sarcoid skin lesions (biopsy), lung infiltrations, a palpable spleen and a negative Wassermann reaction. I have seen two similar cases. The first, a young man of twenty-five was found on routine insurance examination to be almost blind in the right eye due to chronic irido-cyclitis. The other eye was also slightly affected. On being overhauled it was found that he had considerable mediastinal and hilar gland enlargement. All further investigations were unhelpful and his general health was good. The glands settled down over a period of several months but the iritis continued to show slow progress. The second patient had been diagnosed as having a chronic sarcoid skin patch and when I came to examine her I found that she had a slow and quite asymptomatic irido-cyclitis. In both instances there was no gross inflammation, very little keratitis punctata and the posterior 
synechiae were brown and shaggy. These points together with a painless, insidious and relatively benign course may mark it off from other forms of uveitis. It has been suggested that uveoparotid fever may well be a clinical mutation of sarcoidosis though it must be remembered that in some fatal cases miliary tuberculosis has been shown. Krumskal and Levitt (1935) described a case of uveo-parotid fever in which only sarcoidosis could account for all the facts. A woman of 34 showed the positive findings of chronic irido-cyclitis, firm parotid swellings, chronic skin lesions on the legs, an isolated gland in the neck, large glands at the roots of the lungs, an enlarged spleen, pyrexia and negative tuberculin reactions. Section of the gland in the neck showed a hyperplastic epithelioid reaction with giant-celled systems but no caseation and no tubercle bacilli. The authors regard this case as evidence for the tuberculous nature of Heerfordt's syndrome but in future I think that we will have to think of sarcoidosis.

With this kind of case in mind Professor Cohen asked me to see to what extent patients with chronic uveitis were actually suffering from generalised sarcoidosis. It was possible to examine twenty-five cases, all of whom were very chronic and intractable and in whom no cause had been found. No acute cases were included in this group.

\section{Analysis of the Clinical Material}

The patients who form the material for this paper were seen at St. Paul's Eye Hospital, where I was able to go into their history carefully and examine them. They had been in most cases attending the out-patient department for several years. Most had been investigated in hospital for focal sepsis, tuberculin reactions. Wassermann reactions, protein sensitivity, and so on. In addition it was possible to have their chests radiographed for the exclusion of active tuberculosis and sarcoid infiltrations of the glands and lung fields. The details of my findings are in the accompanying tables. Grouping according to age of onset and sex seems to be justified by the closeness of the ages with only one exception in Group II. Though the numbers are small I think that this exact grouping must have some aetiological significance. The average age in Group $I$ is 21 , Group II is 38, and in Group III is 50 .

The general health before and during the illness was uniformly good. In Group III one began to find the chronic degenerative changes so common at that age-obesity, villous arthritis and some hyperpiesia. There was no true rheumatoid arthritis in any patient. In a detailed survey there was no outstanding family ailment. And though these 25 patients subtended a large number of relatives there were no other instances of iritis. The evidence 
ROBERT KEMP

GROUP I-YOUNG WOMEN

\begin{tabular}{r|r|r|l|l|l|l|l} 
No. & Age & Duration & Onset & Course & Extent & Vision & Associated Lesions \\
\hline 1 & 25 & 5 years & Slow & Chronic & Bilat. & Reads & Slight mediast. glands \\
2 & 25 & 5 years & Acute & Recurr. & Bilat. & P.L. & Psoriasis \\
3 & 30 & 14 years & Slow & Recurr. & Bilat. & P.L. & Nil \\
4 & 25 & 5 years & Slow & Chronic & Bilat. & Reads & Mitral stenosis \\
5 & 26 & 2 years & Insid. & Chronic & Bilat. & P.L. & Cervical adenitis \\
6 & 25 & 2 years & Acute & Chronic & Bilat. & Nil & Nil \\
7 & 16 & 9 years & Acute & Chronic & Bilat. & P.L. & B.P.=170/110 \\
8 & 52 & 20 years & Acute & Recurr. & Bilat. & Nil & Glossitis \\
9 & 55 & 37 years & Acute & Recurr. & Bilat. & Nil & Poor physique
\end{tabular}

GRouP II-MIDDLE-AGED MEN

\begin{tabular}{c|c|c|c|l|l|l|l} 
No. & Age & Duration & Onset & Course & Extent & Vision & \multicolumn{1}{|l}{ Associated Lesions } \\
\hline 10 & 40 & 7 years & Insid. & Chronic & Bilat. & Reads & Dyspepsia-no ulcer \\
11 & 53 & 6 years & Acute & Recurr. & Bilat. & Reads & Healed acute nephritis \\
12 & 45 & 6 years & Acute & Recurr. & Bilat. & Reads & Nil \\
13 & 42 & 1 year & Grad. & Chronic & Bilat. & Reads & Nil \\
14 & 36 & 10 years & Acute & Recurr. & Bilat. & Good & 3 small lipomata \\
15 & 45 & 2 years & Grad. & Chronic & Unilat. & Nil R.E & Nil \\
\hline
\end{tabular}

Group III-ELdERLy WOMEN

\begin{tabular}{c|c|c|l|l|l|l|l}
\hline No. & Age & Duration & Onset & Course & Extent & Vision & \multicolumn{1}{|c}{ Associated Lesions } \\
\hline 16 & 52 & 2 years & Grad. & Recurr. & Bilat. & P L. & Nil \\
17 & 55 & 1 year & Acute & Recurr. & Unilat. & Normal & Villous arthritis knees \\
18 & 54 & 10 years & Grad. & Chronic & Bilat. & Reads & Radiol. phthisis \\
& & & & & & B.P. 170/110 \\
19 & 52 & 3 years & Grad. & Insid. & Bilat. & Reads & Very slight mediast. \\
& & & & & & & glands \\
20 & 62 & 5 years & Acute & Slow & Bilat. & P.L. & General health good \\
21 & 37 & 2 years & Insid. & Slow & Bilat. & P.L. & Nodule thyroid \\
22 & 57 & 4 years & Acute & Recov. & Bilat. & Fair & Vision improving \\
23 & 56 & 8 years & Acute & Recurr. & Bilat. & Reads & B.P. 175/120 \\
24 & 55 & 1 year & Acute & Recurr. & Bilat. & Reads & Healthy \\
25 & 58 & 1 year & Insid. & Chronic & Bilat. & P.L. & Nil \\
\hline
\end{tabular}


in favour of a tuberculous aetrology was scanty. Two relatives had died of phthisis - this figure being rather less than one would have expected in any similar group of families. Two brothers of one patient were in a children's sanatorium with suspected but not active abdominal tuberculosis. One girl of Group I had had glands removed from her neck four years previously and they had been regarded on macroscopic examination as tuberculous. There were two firm glands remaining which clinically might have been due to tuberculosis or sarcoidosis. Biopsy was refused. One woman of Group III with a long history of chest symptoms had a good deal of radiological thickening of the lung fields which was thought by the radiologist to be tuberculous. The sputum was not, however, positive. I have ignored the results of the tuberculin tests though it should be noted that in sarcoidosis it is said that the reactions are more frequently negative than in the normal population.

At the time that I saw these patients the illness had been present for many years-in one instance for 37 years. In Group I the average duration was 11 years. In Group II it was 5 years and in Group III approximately 4 years. There seemed undoubtedly to be two distinct methods of progress but whether this meant more than one pathogenesis it was not possible to say. On the one hand there was an initial acute uniocular iritis settling down into a chronic irido-cyclitis. This was followed at a varying period by recurrent attacks in which the other eye was also involved. On the other hand in rather fewer cases the onset was insidious and painless. Sometimes there was no indication of the disease process until a casual covering of the sounder eye revealed the impairment in the other. Deterioration then took place relentlessly over a matter of years. Both these types were equally represented in all three groups of patients.

When one came to examination good general health was found and no symptoms beyond slight pain and failing vision. As might be expected this gradual failure in adults was accompanied by a fair amount of depression and lack of interest with relative failure of adaptation to the new conditions of limited sight. In addition to the general physical overhaul a special search was made for sarcoid lesions, such as skin changes, adenopathy, splenomegaly, and phalangeal deformities together with radiography of the lungs. There were very few positive findings. The cervical adenitis and the radiological phthisis have been mentioned. Two patients had slight mediastinal gland enlargement. One man had three small subcutaneous nodules clinically resembling lipomata. One of these was excised and on section showed that this was the case.

As for treatment these patients had had every conceivable 
measure during the years that they had been attending the hospital. In the first place any focal sepsis found had been eliminated. As well as local mydriatic measures, they had had courses of organic arsenicals, tuberculin, gold preparation, and autogenous and stock vaccines. Together with mild fever therapy and protein shock with T.A.B., milk injections, autohaemotherapy, peptone, and so on. Several patients had had to sacrifice an eye and many had submitted to iridectomy. In passing, the most optimistic therapeutic report in the literature is the electric pyrotherapy reported by Whitney (1935). But despite the best of treatment and supervision the prognosis as shown by this series is poor. Though it must be remembered that I saw no patients who could have been regarded as responding to therapy or making a spontaneous cure. In all but two patients both eyes were affected. And in only one patient was the vision improving. The eyesight of the rest-as I have briefly indicated in the tables-was greatly limited. The least affected were still able to read, others were deprived of their reading but could grope their way about, but three patients were quite blind. From the grouping it is clear that the worst results are seen in Group I. Whereas in Group III process was slower and it was still possible for instance to continue with household duties.

Such was the material that I was able to examine and though the results were not strikingly positive it was possible to draw the following conclusions.

1. The incidence of non-specific chronic irido-cyclitis seems to fall into three age/sex groupings.

2. Apart from this, present day clinical methods are unable to link the illness with any of the known aetiological causes. It may therefore be a disease sui generis.

3. It follows a chronic progressive course, is not amenable to treatment and the visual prognosis is poor.

4. Sarcoidosis is not a cause in the majority of instances.

Acknowledgment. I would like to thank the honorary staff of St. Paul's Hospital for their courtesy and co-operation.

\section{REFERENCES}

ClegG, J. G.-Trans. Ophthal. Soc. U.K., Vol. XLVIII, p. 63, 1928.

Copps, L. A., and McCormick, G.-Wiscon. Med. Jl., Vol. XXXVI, p. 268, 1937.

Doggart, J. H.-Brit. Med. Jl., Vol. I, p. 249, 1926.

Gordon, H.-Proc. Roy. Soc. Med., Vol. XXX, p. 1057, 1937.

Irons, E. E., and Brown, E. V. I.-Jl. Amer. Med. Assoc., Vol. LXXXI, p. 1770, 1923.

Irons, E. E.-Jl. Amer. Med. Assoc., Vol. CIV, p. 1794, 1935.

Krumskal, I. D., and LevitT, J. M.-Amer. Jl. Ophthal., Vol. XVIII, p. 735, 1935.

ScotT, R. B.-Brit. Med. Jl., Vol. II, p. 777, 1938.

Whitney, E. L.-Jl. Amer. Med. Assoc., Vol. CIV, p. 1794, 1935. 